\author{
Military Technical College \\ Kobry El-kobbah, \\ Cairo, Egypt
}

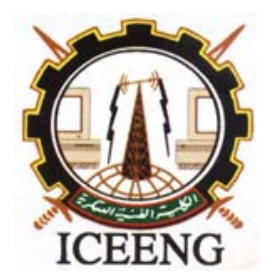

\author{
$5^{\text {th }}$ International Conference \\ on Electrical Engineering \\ ICEENG 2006
}

\title{
PERFORMANCE ANALYSIS OF TRELLIS CODED MODULATION SCHEMES WITH ASYMMETRIC QPSK IN LAND MOBILE SATELLITE FADING CHANNELS
}

Salah EL-Agooz*, Gamal Mabrouk Abdel-Hamid*, and Amru Hassan Hafez*

\begin{abstract}
This paper is concerned with evaluating the performance of trellis coded modulation schemes with asymmetric QPSK when transmitted through a land mobile satellite channel. The channel exhibits fading and shadowing as well as additive white Gaussian noise. Fading and shadowing cause both envelope and phase variations of the received signal. However, it is assumed that the effect of the fading on the phase of received signal is fully compensated using a pilot tone calibration technique. The paper provides an upper bound of pairwise error probability of TCM schemes with both asymmetric and symmetric QPSK modulations. Numerical results show that TCM with asymmetric QPSK provides better performance in terms of bit error probability over TCM with symmetric constellations in Nakagami-m distribution fading channel.
\end{abstract}

\section{KEY WORDS}

Trellis coded modulation, Land mobile satellite fading channel, Asymmetric QPSK constellation.

\section{INTRODUCTION}

Trellis coded modulation (TCM) scheme achieves good power and bandwidth efficiencies for additive white Gaussian noise (AWGN) channel. In the recent years, TCM has increasingly been used in mobile radio and indoor wireless channels, which are often modeled as fading channels. In a bandwidth limited environments, increasing efficiency in frequency utilization can be obtained by choosing higher order modulation schemes however, larger signal power would be needed. Therefore, if a well chosen code can be combined with bandwidth efficient scheme, e.g. Mary PSK, the bandwidth expansion results from coding can be compensated by bandwidth reduction results from the Mary PSK modulation. Furthermore, the coded system allows a reduction in the required signal power by an amount equal to the coding gain, which allows overcoming the increased power required by using the Mary PSK modulation scheme. Performance analysis for trellis-coded M-any phase shift keying (MPSK) and M-any continuous phase frequency shift keying (CPFSK) operating on fading land mobile satellite channel have been presented in [2] and[3]. A complete analysis of TCM for the ideally interleaved Rician fading channel with coherent or differentially coherent detection 
* Egyptian Armed Forces

were reported by Divsalar and Simon In [4] their analysis included derivation of chernoff upper bound on The pairwise error probability. An alternative approach based on a Convolutional code followed by bit interleavers was reported by Zehavi In [5], which yields a better coding gain over a Rayleigh channel with 8-PSK modulation. A series of computer researches has been carried out To obtain good valid trellis codes for 4-any continuous phase frequency Shift keying (CPFSK) with modulation index $h=1 \backslash 2$ in [6] by Pour. He investigated that the criterion for comparison of codes is the maximum free Euclidean distance for a given rate and number of states of the signal space code. A new study for the performance of trellis coded modulation schemes in Rayleigh channel has been introduced in [7]. This technique is based on the derivation of the tight upper expression of the pairwise error probability with the transfer function bounding of each error event path. We also show that for a trellis code that is a linear convolutional code followed by a signal mapper, the number of states may always be reduced from $\mathrm{N}^{2}$ to $\left(\left(\mathrm{N}^{2}-\mathrm{N}\right) / 2\right)+1$ using low complexity techniques is always possible for trellis codes using a linear convolutional encoder have been presented in [8]. The performance of 8-PSK and 8-DPSK trellis codes have been presented for a class of fast fading and mobile satellite communication channels, the fading is assumed Rician shadowed performance has introduced in McLane et al in [9].

In this paper we investigate the performance of TCM with Asymmetric QPSK in mobile satellite channel where fading highly affects the signaling performance. This paper is organized as follows: Section II describes the land mobile channel model. Section III provides analysis of system performance. Section IV shows the numerical results. Section V provides the conclusion.

\section{LAND MOBILE SATELLITE CHANNEL MODEL.}

In this paper we use the channel model given in [10] assuming that, the effect of fading on the phase of received signal is fully compensated using a pilot tone calibration technique. Thus the analysis will focus on the performance degradation due to the effect of fading on the amplitude of the received signal. The fading amplitude of each received channel symbol is described by the Nakagami-m distribution is given by [11].

$P(\rho)=\frac{2 m^{m} \rho^{2 m-1}}{\Gamma(m) \Omega^{m}} \exp \left[-(m / \Omega) \rho^{2}\right], \rho \geq 0$

where

$\Omega=\overline{\rho^{2}}=1$ under normalized conditions,

$\Gamma($.$) is the gamma function, and$

$m=\frac{\left(\overline{\rho^{2}}\right)^{2}}{\left(\rho^{2}-\overline{\rho^{2}}\right)^{2}} \geq 1 / 2$.

\section{ANAYLSIS OF SYSTEM PERFORMANCE}

\section{A. Upper bound on pairwise error probability}

Let $X=\left(x_{1}, x_{2}, x_{3}, \ldots, x_{N}\right)$ represents the transmitted coded symbol sequence of length $\mathrm{N}$, and the corresponding received sequence $Y=\left(x_{1}, x_{2}, x_{3}, \ldots, x_{N}\right)$ is given by

$$
Y=\rho X+N_{n},
$$


where, $\rho$ is a normalized random fading amplitude whose pdf is given by Eq.(1), and $N_{n}$ is a zero mean Gaussian noise random variable with variance $\sigma^{2}$. Assuming infinite interleaving and deinterleaving, so the channel becomes memoryless and the channel transition probability satisfies

$$
P(Y / X ; Z)=\prod_{i=1}^{N} p\left(y_{i} / x_{i} ; z_{i}\right),
$$

where,

$$
Z=\left(z_{1}, Z_{2}, Z_{3}, \ldots, Z_{N}\right) \text { is the channel state information. }
$$

The decoding process uses a metric of the form $\mathrm{m}(\mathrm{Y}, \mathrm{X}$; Z). The total metric of the sequences satisfies the additive property given by the sum of the metrics of each channel input and output pairs of the sequences

$$
\mathrm{m}(\mathrm{Y}, \mathrm{X} ; \mathrm{Z})=\sum_{i=1}^{N} m\left(y_{i}, x_{i} ; z_{i}\right) \text {. }
$$

Using maximum likelihood sequence estimation (MLSE) decoder, the maximum likelihood metric can be written as

$$
\mathrm{m}(\mathrm{Y}, \mathrm{X} ; \mathrm{Z})=\ln \mathrm{P}(\mathrm{Y} / \mathrm{X}, \mathrm{Z}) \text {. }
$$

To find an upper bound on the average bit error probability performance using Viterbi decoder, we first find the pairwise error probability $\mathrm{P}(X \rightarrow \hat{X})$ which represents the probability of choosing the coded sequence $\hat{X}=\left(\hat{x}_{1}, \hat{x}_{2}, \ldots, \hat{X}_{N}\right) \quad$ instead of $X=\left(x_{1}, x_{2}, x_{3}, \ldots, x_{N}\right)$. The probability that decoder decides $\hat{X}$ instead of $\mathrm{X}$ is provided when

$$
\sum_{i=1}^{N} m\left(y_{i}, \hat{x}_{i} ; z_{i}\right) \geq \sum_{i=1}^{N} m\left(y_{i}, x_{i} ; z_{i}\right) .
$$

Thus, the pairwise error probability $\mathrm{P}(X \rightarrow \hat{X})$ is given by:

$$
\mathrm{P}(X \rightarrow \hat{X})=\operatorname{Pr}\left\{\left(\sum_{i=1}^{N} m\left(y_{i}, \hat{x}_{i} ; z_{i}\right) \geq \sum_{i=1}^{N} m\left(y_{i}, x_{i} ; z_{i}\right)\right) / \mathrm{X}\right\} .
$$

By applying the Chernoff bound, so Eq.(7) can be represented as

$$
\mathrm{P}(X \rightarrow \hat{X}) \leq \prod_{i=1}^{N} \mathrm{E}\left\{\exp \left(\lambda\left[\mathrm{m}\left(y_{i}, \hat{x}_{i} ; z_{i}\right)-\mathrm{m}\left(y_{i}, x_{i} ; z_{i}\right)\right]\right) / \mathrm{X}\right\},
$$

where, $\mathrm{E}$ is the statistical average, and $\lambda$ is the Chernoff parameter to be optimized to obtain the tightest possible exponential bound. From Eq.(2), and Eq.(5) we can get

$$
\mathrm{m}\left(y_{i}, x_{i} ; z_{i}\right)=-\left|y_{i}-\rho x_{i}\right|^{2} \text {. }
$$

For constant envelope signal sets e.g. QPSK, and by substituting Eq.(9) into Eq.(8), a conditioned pairwise error probability can be written as

$$
\mathrm{P}(X \rightarrow \hat{X} / \rho) \leq \prod_{i=1}^{N} \exp \left\{-\lambda\left|\mathrm{xi}-\hat{x}_{i}\right| 2\right\}^{*} \mathrm{E}\left\{\exp \left[-2 \lambda \rho \operatorname{Re}\left\{\mathrm{xi}\left(\mathrm{xi}-\hat{x}_{i}\right)^{*}\right]\right\}\right.
$$

Representing the complex noise random variable of $N_{n}$, in terms of its real and imaginary parts where $\operatorname{Re}\left\{N_{n}\right\}$ and $\operatorname{Im}\left\{N_{n}\right\}$ are uncorrelated, zero mean Gaussian random variables each with variance $\sigma_{N}^{2}=\sigma^{2} / 2$. Optimization of Eq.(10) over the Chernoff bound parameter $\lambda$ yields

$$
\lambda_{\text {opt }}=(1 / 4) \sigma_{N}^{2} .
$$

Substituting from Eq.(11) into Eq.(10) we obtain 


$$
P(X \rightarrow \hat{X} / \rho) \leq \exp \left\{\frac{1}{8 \sigma_{N}^{2}} d^{2}(X, \hat{X})\right\},
$$

where,

$$
d^{2}(X \rightarrow \hat{X})=\sum_{i=1}^{N}\left|x_{n}-\hat{x}_{n}\right|^{2},
$$

represents the squared Euclidean distance between the two symbol sequences $\mathrm{X}$ and $\hat{X}$. Rewriting Eq.(12) in terms of the average symbol energy-to-noise spectral density $E_{s} / N_{o}$ and averaging it over the normalized amplitude fading $\rho$ with pdf given Eq.(1), we get

$$
P(X \rightarrow \hat{X}) \leq \prod_{i=1}^{N}\left(1+\frac{\overline{E_{s}}}{4 m N_{0}}\left|x_{i}-\hat{x}_{i}\right|^{2}\right)^{-m} .
$$

For $m \geq 1$, the Nakagami-m distribution is considered as approximation to the Rician distribution which is described as

$$
P(\rho)=\frac{\rho}{\sigma^{2}} \exp (-K) \exp \left(\frac{-\rho^{2}}{2 \sigma^{2}}\right) I_{o}\left(\frac{2 K \rho^{2}}{\sigma^{2}}\right)^{1 / 2} .
$$

where,

$$
K=\frac{\left(m^{2}-m\right)^{1 / 2}}{m-\left(m^{2}-m\right)^{1 / 2}} \quad m \geq 1,
$$

represents the ratio of the power in the scattering (fading) component to the power in the direct (specular) component.

\section{B. Upper bound on bit error probability}

To derive an upper bound of the bit error probability, from the pairwise error probability upper bound Eq.(14) is rewritten as

$$
P(X \rightarrow \hat{X}) \leq \prod_{i=1}^{N} \exp \overline{\left\{-\frac{E_{s}}{4 N_{o}} \Delta^{2}\right\}}=\prod_{i=1}^{N} \overline{Z^{\Delta^{2}}},
$$

where,

$$
\begin{aligned}
& \Delta^{2}=\sum_{i=1}^{N} \rho^{2}\left|x_{i}-\hat{x}_{i}\right|^{2}, \\
& \mathrm{Z}=\exp \left\{-E_{s} / 4 N_{o}\right\},
\end{aligned}
$$

represents the Bhattacharyya parameter, and the overbar denotes averaging over $\rho$. For the case of no fading Eq.(17) is given with no overbar and $\Delta^{2}=\sum_{i=1}^{N}\left|x_{i}-\hat{x}_{i}\right|^{2}$.

The union bound of the probability of an error event is given by [11]:

$$
P_{b} \leq \sum \sum_{X} P(X) \sum_{X, \hat{X} \in C} a(X, \hat{X}) P(X \rightarrow \hat{X}),
$$

where

$a(X, \hat{X})$ is the number of bit errors that occur when the sequence $X$ is transmitted and sequence $\hat{X} \neq X$ is chosen by the decoder, $P(X)$ is the priori probability of transmitting $X$. 
Also, the probability of an error event is upper bounded in terms of the transfer function of the error-state transition diagram, i.e.[12].

$$
P(\{E\}) \leq T(D, L, I) .
$$

where,

$T(D, L, I)$ is the transfer function of the error-state transition diagram.

The average bit error probability upper bound $P_{b}$ for the no fading channel is then given by:

$$
P_{b} \leq\left.\frac{1}{b} \frac{\partial}{\partial I} \bar{T}(D, L, I)\right|_{L=1, D=Z},
$$

where

$\mathrm{b}$ is the number of input bits per trellis interval.

Determination of the encoder transfer function requires a specification of the binary convolutional encoder used by the trellis code.

\section{Bit error Probability of rate 1/2 Trellis coded Asymmetric QPSK}

For rate 1/2 Trellis coded combined with Asymmetric QPSK modulation (TCAQPSK) as illustrated in Fig.2, the system performance can be obtained as follows. From the error-state transition diagram, the transfer function is given by[12] :

$$
T(D, I)=\frac{4 a c}{1-2 b},
$$

where

$$
\begin{aligned}
& \mathrm{a}=\frac{I}{2} D^{4}, \\
& \mathrm{~b}=\frac{I}{2} D^{4 /(1+\alpha)}, \\
& \mathrm{c}=\frac{I}{2} D^{4 \alpha /(1+\alpha)}, \\
& \text { with } \mathrm{D}=\exp \left(-\frac{E_{b}}{4 N_{O}}\right), \\
& \text { and } \alpha=\tan ^{2} \frac{\phi}{2} \text { is the ratio of signal powers between I and } \mathrm{Q} \text { channels. }
\end{aligned}
$$

Substituting from the branch label gains a, b, c into Eq.(23)

$$
T(D, I)=\frac{I D^{4(1+2 \alpha) /(1+\alpha)}}{1-I D^{4 /(1+\alpha)}} .
$$

To take the fading effect into account the error-state transition diagram is modified by replacing $D^{\beta}$ by $D^{\beta \rho 2}$ in the branch label gains a, b, and c respectively where the corresponding values of $\beta$ are given by:

$$
\beta 1=4, \quad \beta 2=4 /(1+\alpha) \text {, and } \beta 3=4 \alpha /(1+\alpha) \text {. }
$$

In case of Rician fading $\overline{D^{\beta \rho 2}}$ is evaluated by averaging it over $\rho$ using the Nakagami-m distribution pdf of Eq.(1)

$$
\overline{D^{\beta \rho 2}}=\left(\frac{1+k}{1+k+\beta \gamma}\right) D^{k \beta(1+k+\beta \gamma)},
$$

where,

$$
\gamma=\frac{\overline{E_{b}}}{4 N_{o}} .
$$


For Rayleigh fading as a special case with $\mathrm{K}=0 \mathrm{Eq} .(26)$ becomes

$$
\overline{D^{\beta \rho 2}}=\frac{1}{1+\beta \gamma} \text {. }
$$

Evaluating $T(D, I)$ of Eq.(23) using Eq.(26), and performing the differentiation required in Eq.(22) we get

$$
\mathrm{P}_{\mathrm{b}} \leq \frac{\xi_{1} \xi_{3} D^{\zeta_{1}+\zeta_{3}}}{\left(1-\xi_{2} D^{\zeta_{2}}\right)^{2}}
$$

where

$$
\begin{aligned}
& \xi_{i}=\frac{1+k}{1+k+\gamma \beta_{i}}, \\
& \zeta_{i}=\frac{\beta_{i} k}{1+k+\gamma \beta_{i}}, \quad \mathrm{i}=1,2,3, \\
& \beta 1=4, \beta 2=4 /(1+\alpha), \beta 3=4 \alpha /(1+\alpha), \\
& \text { and } \gamma=\frac{\overline{E_{b}}}{4 N_{o}} .
\end{aligned}
$$

In case of Rician fading, the bit error probability upper bound for asymmetric QPSK over Rician fading channel is obtained by optimized the asymmetry parameter $\alpha$ which is found to be $\alpha=3.078$. Substituting with $\alpha$,

$$
P_{b} \leq \frac{\left(\frac{K+1}{K+1+\left(\bar{E}_{b} / N_{o}\right)}\right)\left(\frac{K+1}{K+1+0.755\left(\bar{E}_{b} / N_{o}\right)}\right) * D^{\left(\left(\frac{4 * K}{K+1+\left(\bar{E}_{b} / N_{o}\right)}\right)+\left(\frac{3.09 *^{*} K}{K+1+0.755\left(\bar{E}_{b} / N_{o}\right)}\right)\right)}}{\left(1-\frac{K+1}{K+1+0.245\left(\bar{E}_{b} / N_{o}\right)} * D^{\left(\frac{.098 *^{*} K}{K+1+0.245\left(\bar{E}_{b} / N_{o}\right)}\right)}\right)^{2}} .
$$

Similarly, for symmetric QPSK over Rician fading we substitute with $\mathrm{K}=10$ and $\alpha=1$, into Eq.(31) we obtained

$$
P_{b} \leq \frac{\left(\frac{K+1}{K+1+\left(\bar{E}_{b} / N_{o}\right)}\right)\left(\frac{K+1}{K+1+\left(\bar{E}_{b} / 2 N_{o}\right)}\right) * D^{\left(\left(\frac{4^{*} K}{K+1+\left(\bar{E}_{b} / N_{o}\right)}\right)+\left(\frac{2^{*} K}{K+1+\left(\bar{E}_{b} / 2 N_{o}\right)}\right)\right)}}{\left(1-\frac{K+1}{1+K+\left(\bar{E}_{b} / 2 N_{o}\right.} * D^{\left.\left(\frac{2^{*} K}{K+1+\left(\bar{E}_{b} / 2 N_{o}\right)}\right)\right)^{2}}\right.} .
$$

In case of asymmetry Rayleigh fading the optimum parameter is obtained by differentiating Eq.(29) and equating the result to zero. The result is

$$
\alpha=\frac{-4+\bar{E}_{b} / N_{o}\left(\sqrt{17+8 \bar{E}_{b} / N_{o}}-1\right)}{4\left(1+\bar{E}_{b} / N_{o}\right)} .
$$

Substituting from Eq.(33) into Eq.(29) with $\mathrm{K}=0$ we obtain

$$
P_{b} \leq \frac{\left(3+\sqrt{17+8 \bar{E}_{b} / N_{o}}\right)\left[4\left(\bar{E}_{b} / N_{o}\right)^{2}+\left(\bar{E}_{b} / N_{o}\right)\left(7+\sqrt{17+8 \bar{E}_{b} / N_{o}}\right)\right]^{2}}{16\left(\sqrt{17+8 \bar{E}_{b} / N_{o}}-1\right)\left(\bar{E}_{b} / N_{o}\right)^{2}\left(1+\bar{E}_{b} / N_{o}\right)^{4}} .
$$

Similarly, for symmetric QPSK over Rayleigh fading with $\alpha=1$ and $K=0$ in Eq.(29) can be written as 


$$
P_{b} \leq\left(\frac{1+\frac{\overline{E_{b}}}{2 N_{O}}}{\frac{\overline{E_{b}}}{2 N_{O}}}\right)^{2}\left(\frac{1}{1+\frac{\overline{E_{b}}}{N_{O}}}\right)\left(\frac{1}{1+\frac{\overline{E_{b}}}{2 N_{O}}}\right) .
$$

In absence of fading, the bit error probability upper bound for asymmetric QPSK is obtained by optimization over the asymmetry parameter $\alpha$. This can be done by differentiating Eq.(22) with respect $\alpha$ and equating the result to zero. The result is:

$$
\alpha=\frac{E_{b} / N_{o}}{\ln 3}-1
$$

Substituting the value of $\alpha$ from Eq.(36) into Eq.(30) and then substituting into Eq.(29). The result is:

$$
P_{b} \leq \frac{\xi_{1}^{\prime} \xi_{2}^{\prime} \exp \left[-\left(\zeta_{1}^{\prime}+\zeta_{2}^{\prime}\right)\right]}{\left(1-\xi_{2}^{\prime} \exp \left(-\zeta_{2}^{\prime}\right)\right)^{2}}
$$

where

$$
\begin{aligned}
& \zeta_{i}^{\prime}=\frac{\beta_{i}^{\prime} K}{1+K+\beta_{i}^{\prime}} ; \mathrm{i}=1,2,3, \\
& \xi_{i}^{\prime}=\frac{1+K}{\beta_{i}^{\prime}+1+K} ; \mathrm{i}=1,2,3, \\
& \beta_{1}^{\prime}=\frac{\bar{E}_{b}}{N_{o}}, \beta_{2}^{\prime}=\ln 3, \text { and } \beta_{3}^{\prime}=\frac{\bar{E}_{b}}{N_{o}}-\ln 3 .
\end{aligned}
$$

For the purpose of comparison, we use the corresponding upper bounds of uncoded BPSK (same bandwidth as rate $1 / 2$ trellis-coded QPSK) in case of the Rician and Rayleigh fading channels [14].

For Rician fading channel the bit error probability upper bound is given by:

$$
p_{b} \leq \frac{K+1}{K+1+\overline{E_{b}} / N_{0}} \exp \left(-\frac{k \overline{E_{b}} / N_{0}}{K+1+\overline{E_{b}} / N_{0}}\right),
$$

and for case Rayleigh fading channel,

$$
p_{b} \leq \frac{1}{1+\overline{E_{b}} / N_{0}} .
$$




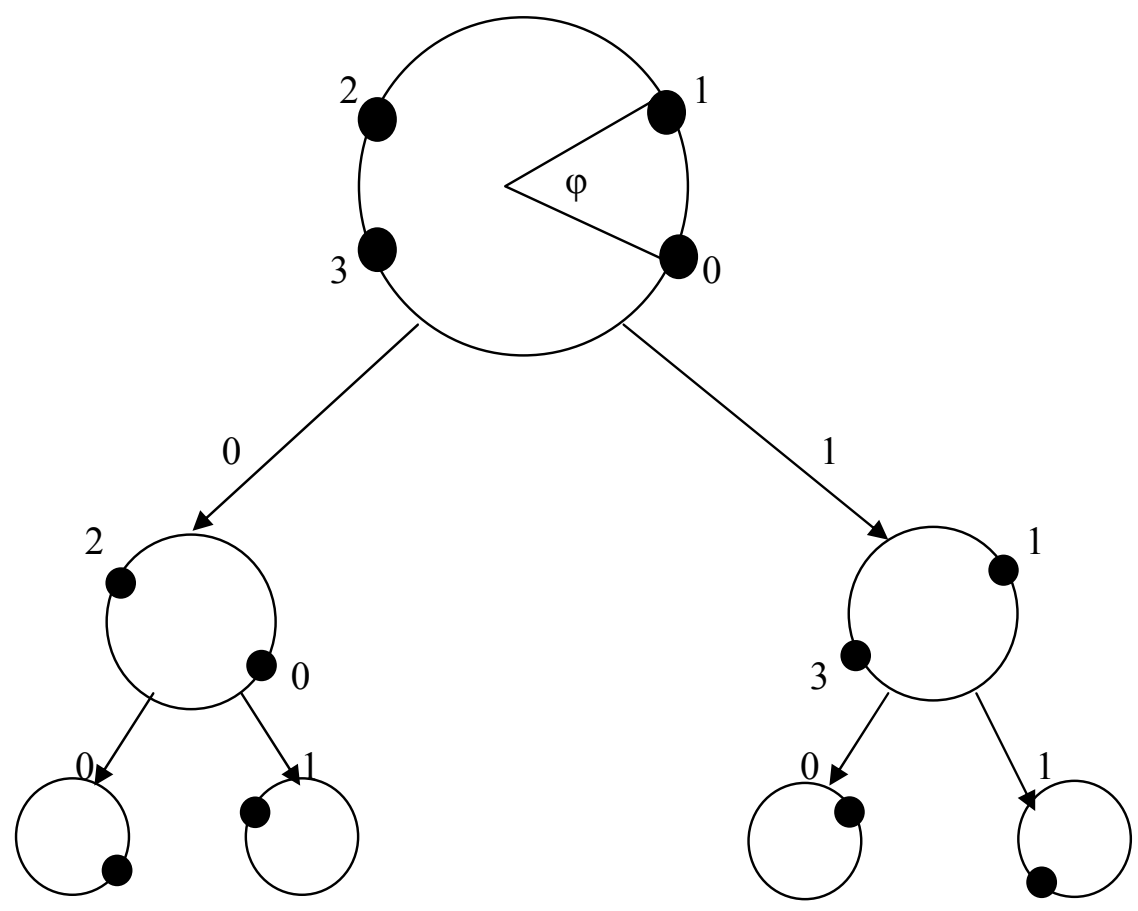

$\mathrm{x} 1 \mathrm{x} 2 \quad 00$

10

01

11

Fig.2.a Set partitioning of asymmetric QPSK

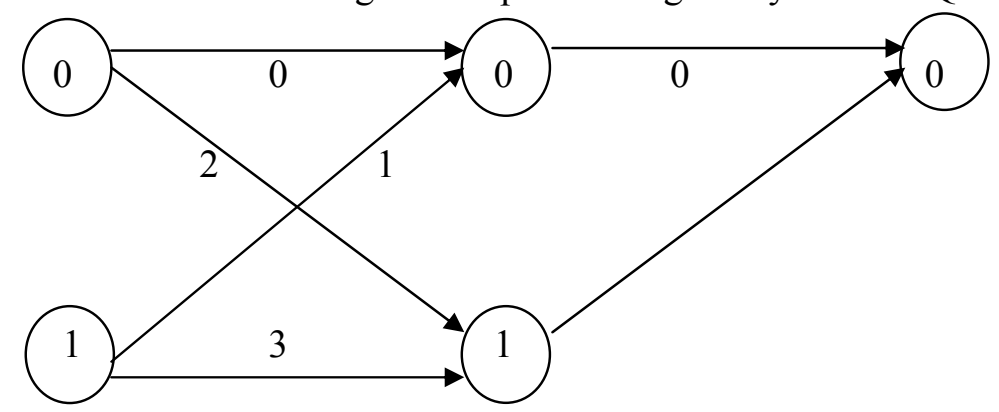

Fig.2.bTrellis diagram and signal assignment for asymmetric QPSK

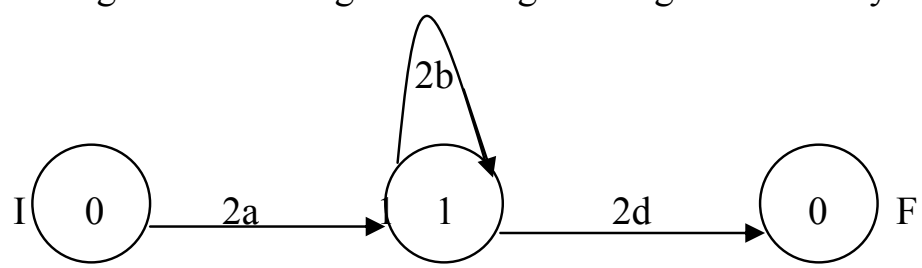

Fig.2.c Error-state transition diagram for trellis diagram of Fig.b .

Fig.2 Rate 1/2 trellis coded asymmetric QPSK

\section{Numerical Results}

The results are obtained by numerically evaluating the bit error probability upper bound given by Eq.(31) to Eq.(39). Fig.1 and Fig. 2 show the BEP for rate 1/2 trellis coded in case of asymmetric and symmetric QPSK versus the signal to noise ratio over Rician fading, respectively. From these figures we can notice that the trellis coded asymmetric and symmetric QPSK provide a better performance compared with uncoded BPSK with Rician fading. Also, from Fig.1, it is clear that the performance of asymmetric QPSK with Rician fading is comparable with the asymmetric QPSK with no fading. Fig.3 and Fig.4 show the 
BEP for rate $1 / 2$ trellis coded asymmetric and symmetric QPSK versus the signal to noise ratio over Rayleigh fading, respectively. From these figures we can notice that the trellis coded asymmetric and symmetric QPSK provide a better performance compared with uncoded BPSK with Rayleigh fading. Fig.5 shows the comparison between the BEP for rate $1 / 2$ trellis coded in case of asymmetric and symmetric QPSK over Rician fading channel. We can notice that trellis coded asymmetric QPSK provide a better performance over symmetric one. Fig.6 shows the comparison between the BEP for rate 1/2 trellis coded asymmetric and symmetric QPSK over Rayleigh fading channel. From Fig.6, it is clear that the trellis coded asymmetric QPSK provides a better performance over a symmetric one.

\section{CONCLUSIONS}

The performance of trellis coded modulation schemes with asymmetric QPSK when transmitted through a land mobile satellite channel has been evaluated. We introduced an upper bound of pairwise error probability of TCM scheme for both asymmetric and symmetric QPSK modulations. The BEP of both asymmetric and symmetric QPSK combined with TCM scheme operating on the land mobile satellite fading channel has been considered. The transfer function of the error- state diagram is used to obtain an upper bound on the bit error probability. The obtained results show that the performance of asymmetric signal constellations combined with TCM provides a better performance over the traditional symmetric constellations combined with TCM in Nakagami-m distribution in land mobile satellite channels.

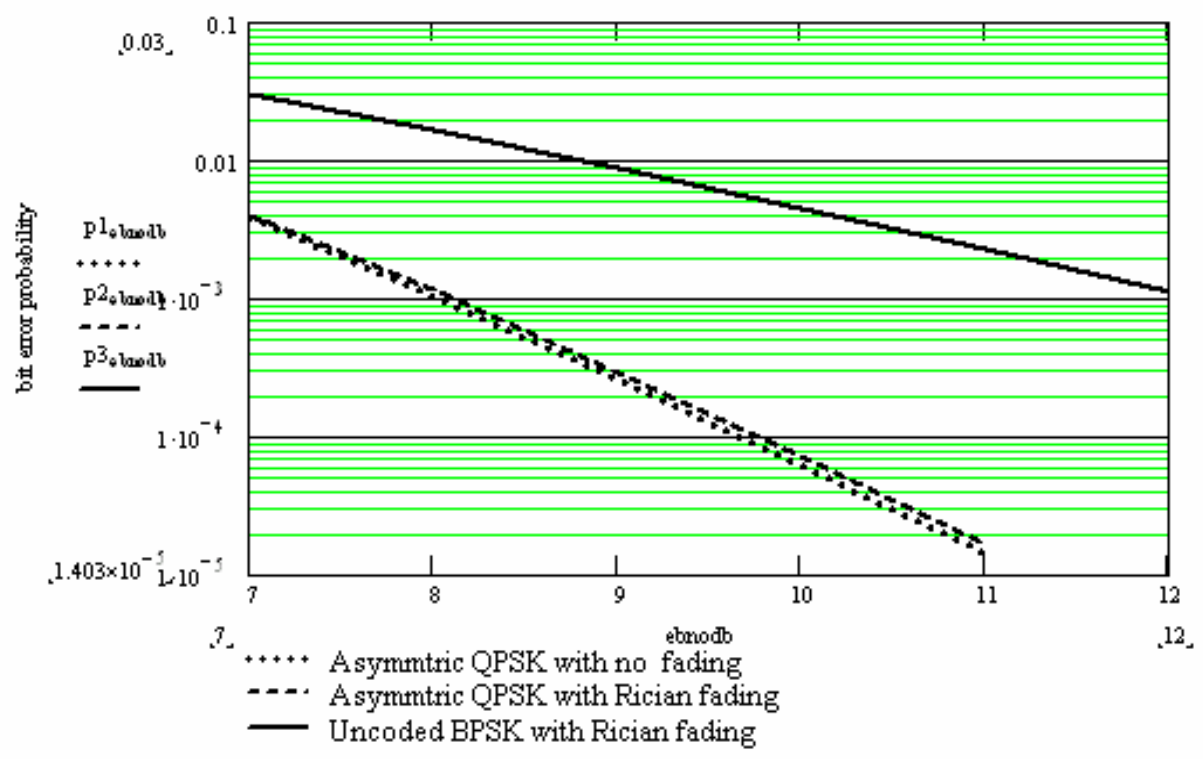

Fig.1 The bit error probability for rate $1 / 2$ trellis coded asymmetric QPSK 


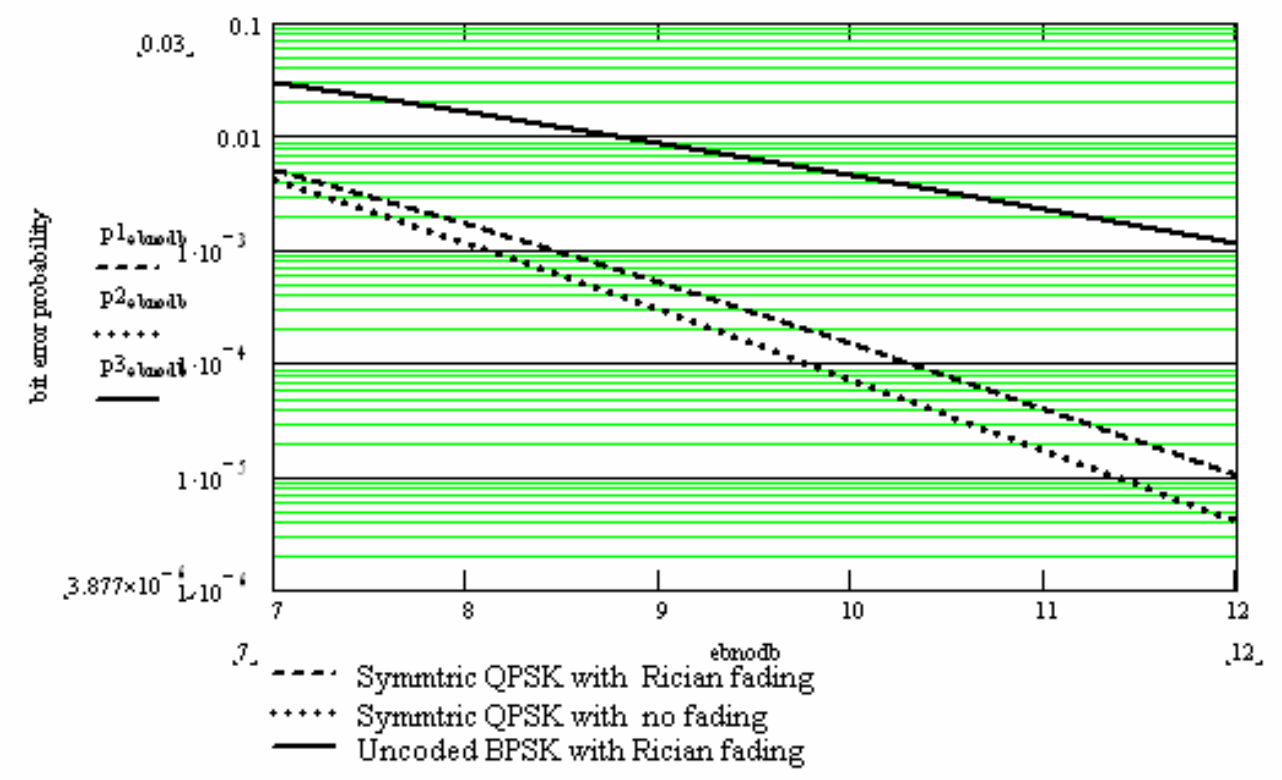

Fig.2 The bit error probability for rate $1 / 2$ trellis coded symmetric QPSK scheme versus $E_{b} / N_{o}$ over the Rician fading channel.

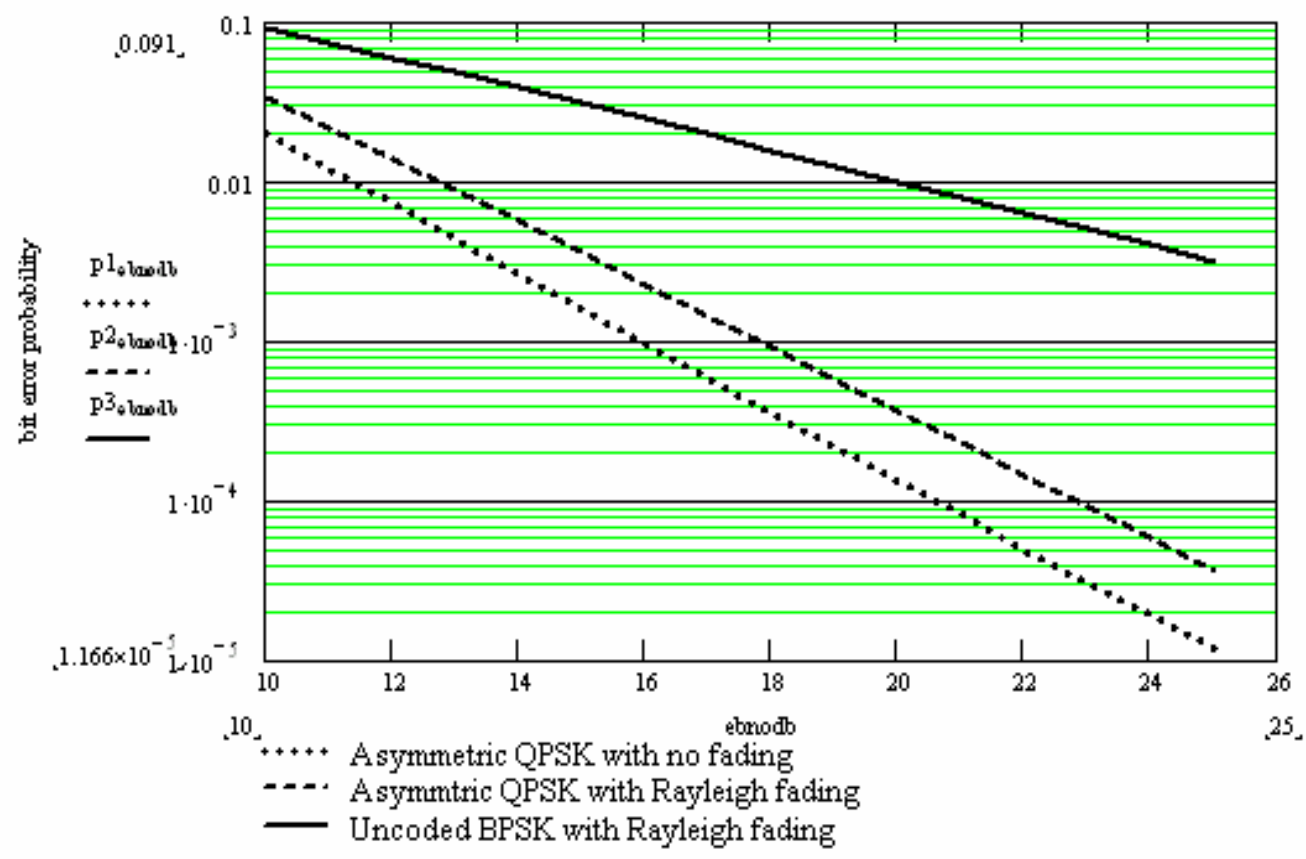

Fig.3 The bit error probability for rate $1 / 2$ trellis coded asymmetric QPSK scheme versus $E_{b} / N_{o}$ over the Rayleigh fading channel. 


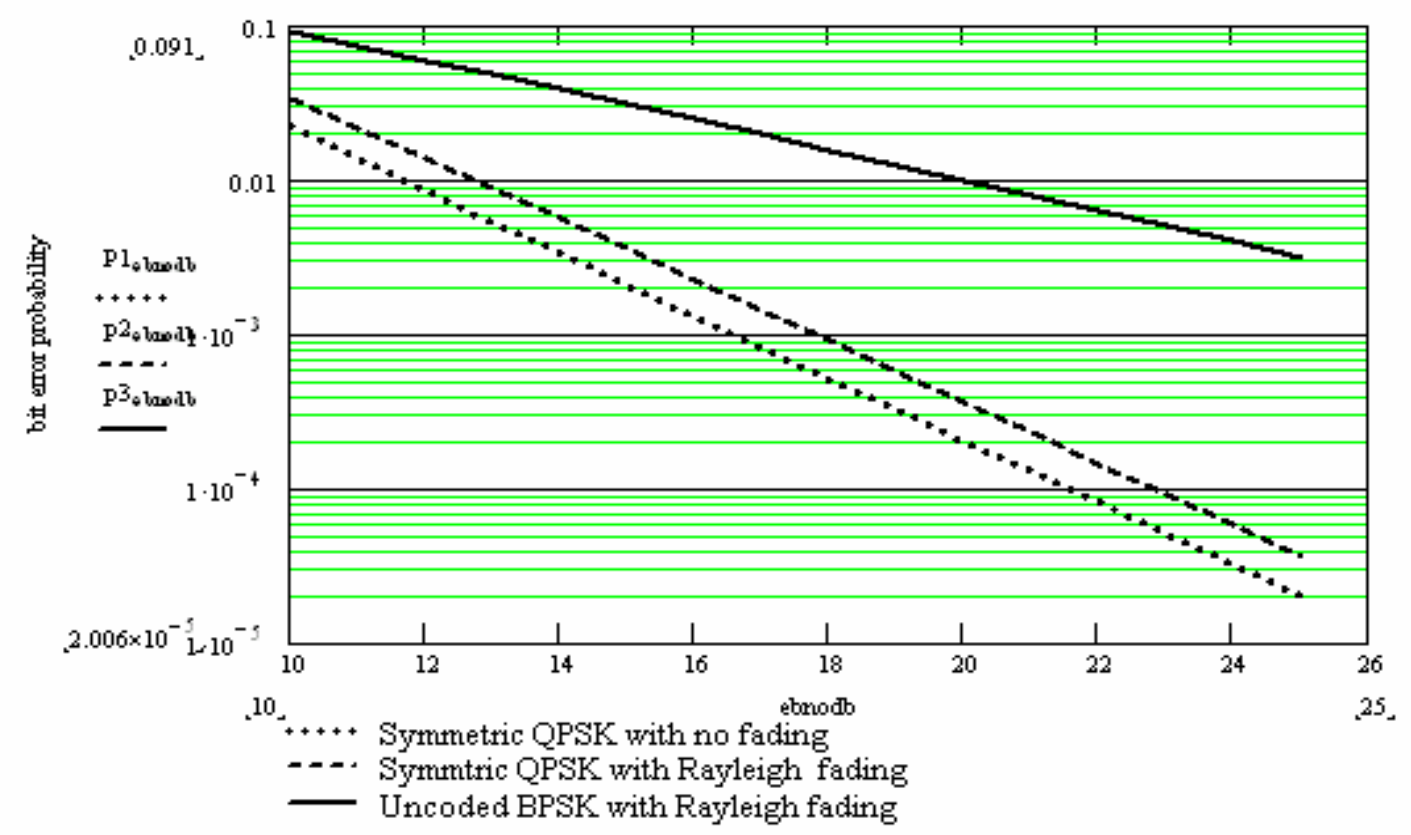

Fig.4 The bit error probability for rate $1 / 2$ trellis coded symmetric QPSK scheme versus $E_{b} / N_{o}$ over the Rayleigh fading channel.

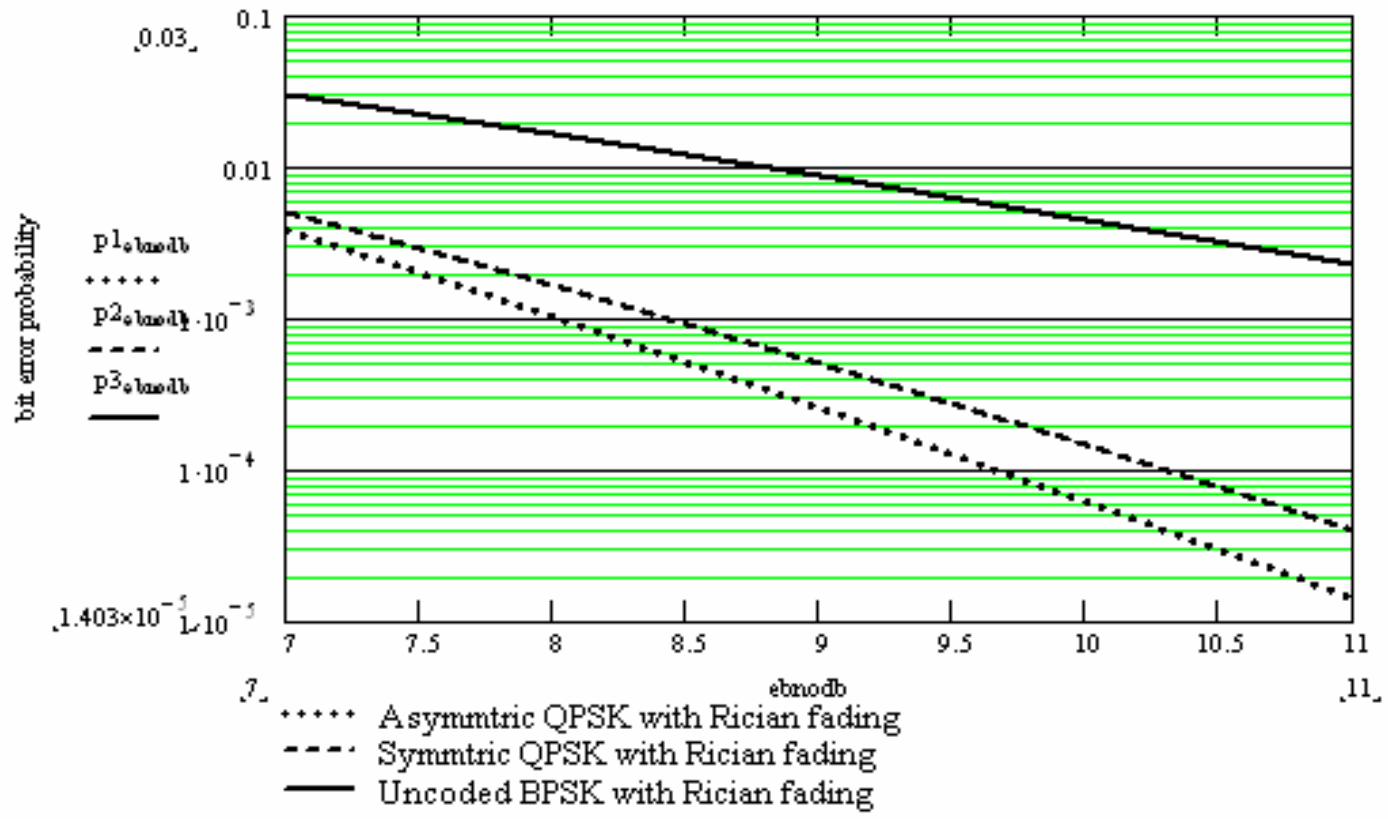

Fig. 5 The bit error probability for rate $1 / 2$ trellis coded asymmetric and symmetric QPSK schemes versus $E_{b} / N_{o}$ on the Rician fading channel. 


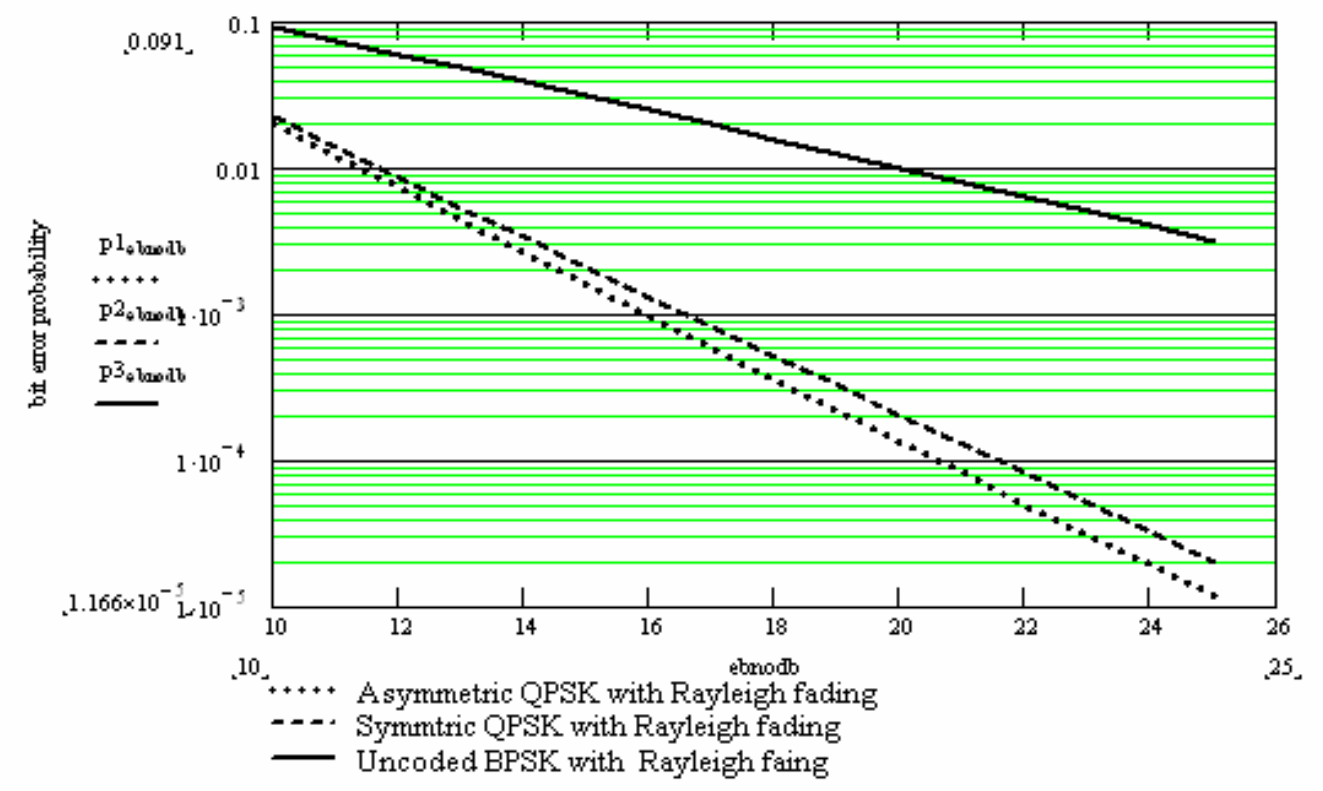

Fig.6 The bit error probability for rate $1 / 2$ trellis coded asymmetric and symmetric QPSK schemes versus $E_{b} / N_{o}$ on the Rayleigh fading channel.

\section{REFERENCES}

[1]Gottfried Ungerboeck, Channel Coding With Multilevel /Phase Signals, IEEE Transactions on Information Theory, Vol.IT-28 , No.1,pp 55-67, January 1982.

[2] Dariush Divsalar and Marvin K.Simon, The Design of Trellis Coded PSK for Fading Channels : Set Partitioning for Optimum Code Design, IEEE Transactions on Communication, Vol.Com-36, No.9, pp 55-67,September 1988.

[3] Bixio Rimoldi, Design of Coded CPFSK Modulation System for Bandwidth and Energy Efficiency, IEEE Transactions on Communication, Vol.Com-37, No.9, pp 897-905, September 1989.

[4] Dariush Divsalar, and Marvin K.Simon, Trellis Coded Modulation for 4800-9600 bits/s Transmission over a Fading Mobile Satellite Channels, IEEE Transactions on Selected Areas In Communication, Vol.SAC-5, No.2, pp 162-175, February 1987.

[5] Ephraim Zehavi, 8-psk Trellis Coded for a Rayleigh Channel, IEEE Transactions on Communication, Vol.Com-40, No 5, pp 873-883, May 1992.

[6] Morteza Naraghi-Pour, Trellis Coded for 4- ary Continuous Phase Frequency Shift Keying, IEEE Transactions on Communication, Vol.com- 41, No .11,pp 1582-1587, November 1993.

[7] Slimane Ben Slimane, and Le-Ngoc, Tight Bounds on the Error Probability of Coded Modulation Schemes in Rayleigh Fading Channels, IEEE Transactions on Vehicular Technology, Vol.44, No.1, pp121-129, February 1995.

[8] Jun Shi, and Richard D.Wesel, Efficient Computation of Trellis Code Generating Functions, IEEE Transactions on Communication VOL.52, No. 2, February 2004. 
[9] P.J.Mclane, P.H. Wittke, and C.Loo, PSK and DPSK Trellis Coded for Fast Fading Shadowed Mobile Satellite Communication Channels, IEEE Transactions on Communication, Vol.Com-36,No.11, pp1242-1246, November 1988.

[10] C.Loo, Measurement and Models of a land mobile satellite channel and their Application to MSK signals, IEEE Transactions on Vehicular Technology, Vol.36, No.1, pp 114-121, August 1987.

[11] Shaini S. Periyalwar and Solomon M, Multiple of Trellis Coded Frequency and Phase Modulation, IEEE Transactions on Communication, Vol.40,No.6, June 1992.

[12] A.J.Viterbi and J.K.Omura, Principles of Digital Communication and Coding, McGrawHill, New York, 1987.

[13]Ezio Biglieri, Dariush Divsalar, Peter J.Mclane, and Marvin K.Simon, Intoduction to Trellis Coded Modulation with Applications, Macmillan Company, New York, 1991.

[14] Venkata Subramaniam, B. Sundar Rajan, and Rajendar Bahl, ,Performance of 4- and 8State TCM Schemes with Asymmetric 8-PSK in Fading Channels, IEEE Transactions on Vehicular Technology, vol. 49, no. 1, January 2000. 